\title{
EL POTENCIAL DESARROLLO TURÍSTICO INTEGRAL DE LOS MUNICIPIOS DEL ÁREA DEL ENTORNO DEL AEROPUERTO DE CASTELLÓ 1
}

\author{
Diego López Olivares \\ Juan Bautista Ferreres Bonfill \\ Ouafae Abdelouahab Reddam \\ Obdulia Monteserín Abella \\ Universidad Jaume I. Castellón
}

\section{RESUMEN}

Los espacios rurales de interior del arco mediterráneo occidental, aún presentan síntomas de las crisis de los modelos agropecuarios de la mitad del siglo pasado. Sin embargo, a pesar de estas debilidades la sociedad civil de estas áreas intenta aprovechar las nuevas dinámicas socio-económicas, entre ellas las vinculadas a la actividad turística. Al respecto, esta investigación se centra en el área territorial del entorno del aeropuerto de Castellón, constituida por nueve municipios, Benlloch, Borriol, Cabanes, la Pobla Tornesa, Sant Joan de Moró, la Torre d'en Doménec, Vall d'Alba, Vilafamés y Vilanova d'Alcolea (Municipios Zona Aeropuerto de Castellón MUZAC), pertenecientes a la comarca de La Plana Alta de Castelón (Comunitat Valenciana). En este estudio se aplica una metodología de carácter turístico integral contemplando las fases de análisis, diagnóstico y diseño de estrategias. Significar el carácter participativo público-privado de la investigación desarrollando un cuestionario tipo Delphi, Likert 5, cuyo resultado, junto con el análisis de los elementos del sistema turístico, se han plasmado en una matriz DAFO que posteriormente nos ha facilitado las propuestas de desarrollo turístico por medio de estrategias sostenibles.

Palabras clave: Turismo y desarrollo integral; sistema turístico; productos; participación público-privada; sostenibilidad.

Fecha de recepción: 16 de marzo de 2018.

Fecha de aceptación: 4 de junio de 2018.

Departamento de Historia, Geografía y Arte. Facultad de Ciencias Humanas y Sociales. Universidad Jaume I. Av. Sos Baynat, s/n. 12071 CASTELLÓN DE LA PLANA (España).E-mail: dlopez@uji.es, ferreres@uji.es, o_ben28@hotmail.com.,monteser@uji.es

1 El artículo forma parte del proyecto "Fomento de la investigación y la innovación en el sector turístico valenciano. Análisis, diagnóstico y prognosis del turismo en los municipios del entorno del aeropuerto de Castelló" financiado durante 2016 por la Agència Valenciana del Turisme, siendo el I.P. Diego López Olivares. 


\title{
The integrated tourism development potential of the municipalities the area around Castellón Airport
}

\begin{abstract}
The inland rural areas of the Western Mediterranean arc still show symptoms of the crisis of the agricultural models of the middle of the last century. However, despite these weaknesses, civil society in these areas is trying to take advantage of the new socioeconomic dynamics, including those linked to tourism. In this respect, this research focuses on the territorial area around Castellón Airport consisting of nine municipalities: Benlloch, Borriol, Cabanes, la Pobla Tornesa, Sant Joan de Moró, la Torre d'en Doménec, Vall d'Alba, Vilafamés and Vilanova d'Alcolea (Municipalities of the Castellón Airport Zone - MUZAC), belonging to the county of La Plana Alta de Castellón (Valencian region). In this study, an integrated tourism methodology is applied, including the analysis, diagnosis and strategy design phases. We should highlight the public-private nature of the research, developing a Delphi-type Likert 5 questionnaire. Its results, together with an analysis of the elements of the tourism system, have been fed into a SWOT matrix which has provided us with tourism development proposals through sustainable strategies.
\end{abstract}

Keywords: Tourism and integrated development; tourist system; products; publicprivate participation; sustainability.

\section{INTRODUCCIÓN}

En las áreas rurales de interior del arco mediterráneo occidental persisten aún los síntomas de las crisis de los modelos agropecuarios de la mitad del siglo pasado, reflejándose su repercusión en los recursos territoriales y la socio-economía, entre otros. En esta situación ha incidido el hecho que esos modelos no han sido reemplazados por otros que hayan favorecido el reposicionamiento de las comunidades y la dinamización del espacio. Por lo tanto es frágil la capacidad de respuesta que actualmente presentan las actividades locales tradicionales para reorientar el territorio desde una perspectiva funcional. Sin embargo, a pesar de esta fragilidad territorial, la sociedad civil de esas áreas intenta aprovechar las nuevas dinámicas socio-económicas, entre ellas las vinculadas a la actividad turística, que se desarrollan en el marco de una sociedad más globalizada que ha generado una nueva relación "campo-ciudad" cada vez más imbricada (Manero, 1997; López, 2003).

A pesar de esta tendencia, es cierto que hasta el momento los resultados no han sido los esperados. En ello ha incidido la espontaneidad del desarrollo, la escasa estructuración de la oferta, al igual que la débil incorporación de las nuevas tecnologías en base a las tendencias de los mercados. Tampoco incide positivamente el hecho de considerar a menudo la actividad turística como una alternativa fácil, y no como un instrumento facilitador de un nuevo marco de desarrollo, como lo reflejan las medidas y acciones contempladas en las iniciativas Leader, Proder y Ruralter de la Unión Europea (López, 1999 y 2014). De ahí que las iniciativas turísticas precisen de modelos y metodolo- 
gías científicas, que, evidentemente, adecuándose a la especificidad de los diferentes territorios y a la nueva noción del desarrollo, entendido éste como un proceso de transformación social (Mella, 1998), faciliten la generación de nuevas estructuras de dinamización en las áreas rurales de interior. Es por lo tanto, bajo el prisma de estas reflexiones, que hemos articulado la presente investigación, planteando en primer lugar los aspectos metodológicos del proceso "sistémico integrado turístico", al que le seguirá un diagnóstico turístico integrado y una propuesta de estrategias que faciliten la puesta en valor del potencial turístico del área de estudio.

Así, la investigación se centra en el área territorial del entorno del Aeropuerto de Castellón constituida por nueve municipios -Benlloch, Borriol, Cabanes, la Pobla Tornesa, Sant Joan de Moró, la Torre d'en Doménec, Vall d'Alba, Vilafamés y Vilanova d'Alcolea- que designaremos como MUZAC (Municipios de la Zona del Aeropuerto de Castellón), siendo su extensión de $486,1 \mathrm{~km}^{2}$ y que pertenecen a la comarca de la Plana Alta interior de la provincia de Castellón y se encuentran incluidos en el espacio 3 del Plan de Espacios Turísticos de la Comunitat Valenciana (Figura 1). Los citados municipios además reúnen criterios de homogeneidad tanto en términos de equilibrio territorial, fisiografía y características históricas, como de modalidad turística, oferta de recursos turísticos compartidos y grado de planificación y desarrollo turístico.

\section{Figura 1 \\ DELIMITACIÓN DEL ÁREA DE ESTUDIO MUZAC}

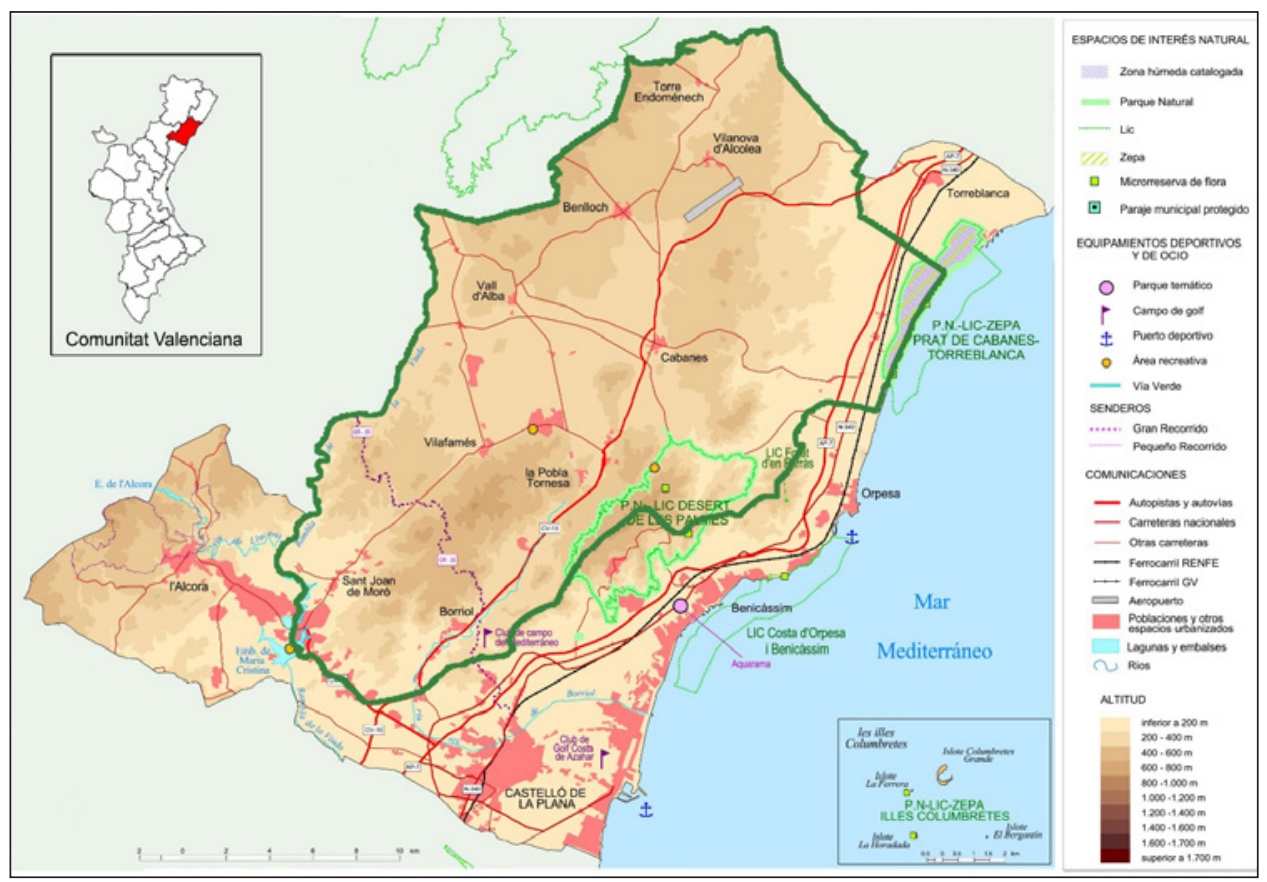

Fuente: Plan de Espacios Turísticos de la Comunitat Valenciana y E.P. 
El aeropuerto se localiza en el interior de la provincia, en los términos municipales de Benlloch y Vilanova d'Alcolea, a $40 \mathrm{~km}$ de la ciudad de Castellón de la Plana, con proximidad a destinos turísticos consolidados del litoral, como Benicàssim, Oropesa o Peñíscola, y del interior como Morella, e industriales y agrícolas como Vila-real, Onda y Borriana. Son destacables algunas de sus características que generan articulación al espacio de su entorno, entre éstas cuenta con buena accesibilidad terrestre a través de la autovía CV-10; y la línea ferroviaria de alta velocidad que une Castellón con Madrid.

Por lo tanto, la delimitación del área de estudio se justifica en primer lugar por la puesta en funcionamiento reciente del aeropuerto, que en septiembre de 2015 recibió el primer vuelo comercial; y por otro en la posibilidad de generar un escenario favorable para el desarrollo turístico, que aproveche la potencialidad del territorio en base a los recursos endógenos.

\section{OBJETIVOS Y METODOLOGÍA}

El objetivo principal de esta investigación es aplicar el modelo de desarrollo turístico integrado, respondiendo los objetivos específicos al análisis de los aspectos generales y de los elementos del sistema turístico, concretándose en:

- La transformación de los recursos endógenos en productos turísticos, integrando las empresas y organizaciones vinculadas con la actividad turística.

- Fortalecer las interrelaciones de los productos con la demanda turística apoyándose en la formación y la aplicación de las nuevas tecnologías.

- Contemplar la tranversalización de la sostenibilidad en el desarrollo turístico.

En los espacios de interior, desarrollar el turismo desde una perspectiva integradora facilita que se conforme en un instrumento capaz de generar dinámicas socioeconómicas, dadas la complejidad y transversalidad que presenta esta actividad en el territorio. De ahí, que se entre en un planteamiento conceptual en el cual el turismo se presenta como un instrumento de desarrollo, más que como un objetivo en sí mismo, ya que la débil situación socio-económica derivada de la decadencia de las estructuras agropecuarias junto al buen posicionamiento del turismo y los servicios puede ayudar a generar sinergias en otras actividades que en alguna medida forman parte de la misma actividad turística (López, 2014). Por ello, esta investigación de carácter turístico integrado contempla tres fases, una de análisis, a la que le sigue una fase de diagnóstico turístico integrado y una tercera fase de diseño de estrategias.

Para el desarrollo de la primera fase, se ha realizado un análisis previo de los aspectos generales del medio físico-natural, la demografía y socio-economía del área de estudio, al que le ha seguido un análisis de los elementos del sistema turístico, que conforman los recursos turísticos, las empresas, la demanda, las infraestructuras y servicios.

En este sentido, la elaboración del inventario de recursos turísticos ha constituido el instrumento básico para su posterior análisis y evaluación, a partir del cual se han identificado, cuantificado y jerarquizado los recursos turísticos de los nueve municipios 
del área de estudio. Los recursos se han categorizado en: A-Recurso natural o paisajístico; B-Recurso histórico-monumental, técnico, etnológico y artístico; C-Artesanales y gastronómicos; y D-Folclore, fiestas, acontecimientos programados (eventos), recursos intangibles y referenciales. Desde una perspectiva más cualitativa, en relación a la potencialidad turística de los recursos se han establecido cinco jerarquías escaladas de 1 a 5. Una vez determinada la jerarquía de cada recurso turístico su evaluación ha concluido con el cálculo del valor jerárquico, que representa la potencialidad turística del recurso (López, 2014).

El análisis de las empresas y organizaciones relacionadas con la actividad turística se ha plasmado en la estructura del alojamiento comercial, es decir, tanto hotelero como extrahotelero, analizando desde una perspectiva cuantitativa y cualitativa su evolución reciente, considerando las fuentes estadísticas oficiales de la Agència Valenciana del Turisme. El análisis se ha completado con la tasa de función turístico-residencial, al ser un indicador importante a determinar, ya que representa el potencial de alojamiento no comercial del área de estudio, y además permite conocer el grado de especialización de un determinado espacio de destino derivado del desarrollo residencial vinculado a la actividad turística. Por último, se contempló el papel que juegan las nuevas tecnologías y la gestión de la información como ventaja competitiva.

El análisis de la demanda se ha efectuado en primer lugar a través de la explotación de los datos estadísticos proporcionados por la oficina de turismo de Vilafamés correspondientes a los años 2015 y 2016; y el estudio realizado por la Agència Valenciana de Turisme a finales de 2015, sobre el perfil del turista internacional que visita la Comunitat Valenciana vía aeropuerto de Castellón.

Para el estudio de las infraestructuras y los servicios, se han aprovechado los resultados de un cuestionario aplicado a través del método tipo Delphi, que como a continuación se detallará, se ha implementado también al resto de elementos del sistema turístico. La utilidad de esta metodología estriba en que nos permite conocer las potencialidades de los elementos del sistema turístico del área de estudio, y su prospectiva, ha ayudado a plantear estrategias de desarrollo con un enfoque integral (Moutinho y Witt, 1995; Linstone, y Turoff, 2002; Garrod y Fyall, 2005; Northcote, Lee, Chok y Wegner, 2008; Solsona y López, 2012; Pulido y Pulido, 2016).

Así, una vez realizado el análisis de los elementos del sistema turístico, se ha diseñado y aplicado un cuestionario a expertos en el sector turístico y responsables de la gestión local del área de estudio a través, como se ha indicado, del método tipo Delphi. La muestra la conformaron 36 cuestionarios distribuidos de manera presencial y online. El cuestionario incluye un total de 102 ítems de escala, basados en la valoración Likert 5 y se estructura en dos bloques de preguntas cerradas y un bloque de preguntas abiertas. El primer bloque de preguntas cerradas se dirigió a los elementos del sistema turístico y lo han configurado siete preguntas, desagregadas en subpreguntas e ítems, que se corresponden a los recursos turísticos; los factores socioeconómicos y medioambientales; la oferta turística; la demanda turística; las infraestructuras y servicios; la promoción, comercialización, financiación y gestión pública; y las nuevas tecnologías y la gestión de la información. El segundo bloque de preguntas cerradas ha sido de 
prospectiva turística y se han dedicado tres preguntas. La primera a valorar los factores que podían representar un hándicap para la evolución turística del MUZAC, la segunda a las acciones que se consideran fundamentales para activar el desarrollo turístico; y la tercera, sobre la sostenibilidad del desarrollo turístico.

En el bloque de preguntas abiertas se han incorporado cinco acerca de las nuevas tendencias y hábitos de la demanda; los obstáculos y medidas para el desarrollo turístico; la identificación de los problemas de planificación turística y si existen vacíos legales; acerca de cómo ven su grado de competitividad turística frente a sus competidores próximos, y por último, una pregunta de carácter general sobre aspectos para el futuro desarrollo turístico.

Además del análisis de los componentes del sistema turístico por medio de la observación indirecta y del trabajo de campo, y las entrevistas a través de los cuestionarios a expertos, se aplican otras técnicas cualitativas como fue el trabajo de participación social, con la realización de dos jornadas de sensibilización -Jornada I celebrada el 22 de julio de 2016 en Benlloch y la Jornada II, el 11 de noviembre de 2016 en Cabanes-.

De este modo el resultado de la fase de análisis de los aspectos básicos y de los elementos del sistema turístico, y los resultados de los cuestionarios al panel de expertos, nos ha permitido elaborar la segunda fase de la investigación, el diagnóstico turístico integrado, el cual se sistematiza en una matriz a través de la técnica DAFO.

Esta matriz constituye el marco para la definición de acciones, que en la tercera fase han sido acometidas a través del diseño de estrategias, articuladas en programas consensuados por los agentes sociales para el desarrollo turístico de los municipios del entorno del aeropuerto de Castellón.

\section{APLICACIÓN DE LA METODOLOGÍA INTEGRADA A LA ZONA MUZAC}

\subsection{Aspectos generales}

El área de estudio responde a una morfología que según criterios litológicos y tectónicos, se inserta en la vertiente mediterránea de la Cordillera Ibérica. En ella se identifican una serie de unidades morfoestructurals, siendo la que le otorga mayor representatividad territorial la conformada por los corredores prelitorales y el Pla de l'Arc, donde se ubican los nueve municipios, y que responde a áreas de acumulación que se corresponde a fosas de hundimiento, con una orientación paralela al mar y que articulan el territorio; el resalte montañoso del Desert de les Palmes, ocupa el extremo más oriental del ramal aragonés de la Cordillera Ibérica, si bien su estructura se halla descompuesta por una densa red de fallas de orientación catalana, es decir NE-SW, que originaron la actual sucesión de resaltes mesozoicos y depresiones internas paralelas al mar; y en la costa la Ribera de Cabanes conformada por dos unidades, el área del Prat, que se caracteriza por ser una costa baja conformada por una restinga de cantos y gravas que cierra el Parque Natural del Prat de Cabanes-Torreblanca, y el núcleo de Torre de la Sal, en donde la restinga de cantos se ve interrumpida por una formación de playa arenosa enmarcada al norte por dunas fósiles, provocando que la línea de costa se adentre y constituya el punto de mayor inflexión del Golfo de Valencia. 
El segundo elemento del medio físico-natural corresponde al clima, puesto que se considera un factor esencial de localización y atracción de la actividad turística. Así para el análisis de los principales elementos del clima y del confort climático se ha tomado como referencia el municipio de Cabanes, por ocupar una posición central en el área de estudio. Si bien se caracteriza por las inversiones térmicas, el comportamiento de las temperaturas medias desprende valores que no descienden de $\operatorname{los} 7,5^{\circ} \mathrm{C}$, siendo éstas superiores a $17^{\circ} \mathrm{C}$ entre mayo y septiembre, encontrándose este valor en el umbral inferior de confort climático para la práctica de actividades al aire libre entre 16 y $18^{\circ} \mathrm{C}$ (Besancenot, 1991). La evolución anual de las precipitaciones responde a la variabilidad pluviométrica y la escasez. Así, en el confort climático incide la disposición del relieve en alineaciones montañosas paralelas y próximas al mar, que originan en este corredor prelitoral fenómenos de inversión térmica (Mateu, 1982) manifestados en bancos de niebla o en heladas, reflejándose diferencias de 2 a $5^{\circ} \mathrm{C}$ entre la cima del Bartolo en el Desert de les Palmes y el Arco Romano de Cabanes.

En cuanto al comportamiento demográfico la zona MUZAC cuenta en 2016 con un conjunto de población de 19.007 habitantes, aunque con una contrastada distribución territorial. En este sentido, aunque en municipios como la Torre d'en Doménc y Vilanova d'Alcolea, la estructura poblacional es más envejecida, en el conjunto destaca el crecimiento natural positivo y la transformación favorable en los grupos de edad entre 30 y 55 años debido al fenómeno de inmigración, ligado al sector cerámico y de la construcción, incrementándose la población un 48,96\% entre 1996 y 2015 que en el caso particular de Borriol está muy ligado a su función de núcleo dormitorio vinculada al área de Castellón de la Plana.

El estudio de la socioeconomía se ha realizado a través del análisis de la especialización productiva de los municipios, reflejado en la distribución de actividades, tanto en el número de empresas como de trabajadores. Los resultados ponen de manifiesto las diferencias del área de estudio con la media provincial que se observa en primer lugar en el porcentaje que representa la industria en el conjunto MUZAC $(54,27 \%)$ frente al $19,08 \%$ a nivel provincial (SEPE, 2016). También se registran diferencias en el sector servicios, pero en este caso MUZAC ofrece un resultado considerablemente inferior $(34,48 \%)$ a la media provincial $(66,44 \%)$, siendo un sector infrarrepresentado. Esta disparidad es especialmente importante desde el punto de vista de la actividad turística.

A escala local, la industria constituye la principal actividad en aquellos municipios con una presencia consolidada de la industria cerámica -Borriol, la Pobla Tornesa, Sant Joan de Moró, Vall d'Alba y Vilafamés. El peso de la ocupación en actividades relacionadas con el turismo -actividades vinculadas con hostelería, comercio minorista, agencias de viaje y empresas de mantenimiento- sobre el total del sector servicios muestra, a excepción de Cabanes, en donde la ocupación en actividades relacionadas con el turismo supera ligeramente el $50 \%$ sobre el total de servicios y más del $25 \%$ sobre el total de actividades, que en los restantes municipios existe una débil presencia de empleo vinculado a este tipo de actividades, en donde incide el hecho de albergar un entorno productivo con alta presencia del sector industrial. 


\subsection{Análisis de los elementos del sistema turístico del área de estudio}

\section{a) Los recursos turísticos}

La elaboración del inventario ha permitido identificar un total de 178 recursos turísticos, los cuales, distribuidos por categorías ofrecen la siguiente distribución: A. Atractivo o recurso natural-paisajístico, a esta categoría pertenecen 45 recursos entre los que sobresalen los espacios naturales protegidos, expresados en los parques naturales del Desert de les Palmes y del Prat de Cabanes-Torreblanca y los parajes naturales municipales, y las playas de la Ribera de Cabanes. Sin embargo, es la categoría B. Recursos histórico-monumentales, técnicos, etnológicos y artísticos, la que ostenta el mayor peso cuantitativo con un total de 81 recursos. Dentro de esta categoría sobresale el patrimonio histórico, artístico y monumental de Vilafamés, el trazado de la Vía Augusta como eje articulador del territorio o el arco romano de Cabanes. La categoría C. Recursos artesanos y gastronómicos, es la que registra menor representatividad, con un total de 13 recursos turísticos, vinculados a la potencialidad de los productos agroalimentarios relacionados con el cultivo de la vid y el almendro. Por último, la categoría D. Folklore, fiestas, acontecimientos programados y recursos intangibles y referenciales, acoge 39 recursos, en donde sobresalen los acontecimientos programados de carácter cultural y deportivo.

Una vez distribuidos los recursos por categorías se ha realizado el análisis cualitativo, al objeto de establecer la jerarquía de los recursos turísticos, es decir, su grado de atracción turística, y a continuación fijar su valor jerárquico, que determina la potencialidad turística del recurso (López, 2014). Así, por categorías de recursos, (Cuadro 1) la categoría A acoge el 31,9\% del total del valor jerárquico. En esta se han establecido 10 recursos de jerarquía 1 que ofrecen el mismo valor jerárquico; y 27 recursos de jerarquía 2 que representan un valor jerárquico de 108. Por su parte, la jerarquía 3 acoge 3 recursos con un valor jerárquico de 27 que se corresponden con el paisaje del Pla de l'Arc y los parajes naturales de El Mollet de Sant Joan de Moró y de Sant Miquel de Vilafamés. No obstante, sobresale el valor jerárquico de la jerarquía 4 que pese a contar con 4 recursos, estos representan un valor de 64 que se corresponden a los espacios naturales protegidos del Desert de les Palmes y del Prat de Cabanes-Torreblanca, y a las playas de cantos rodados del Cudolar y del Quarter Vell. En relación con la jerarquía 5, se halla expresada en la playa de arena fina de Torre la Sal de la Ribera de Cabanes.

La categoría B acoge 30 recursos de jerarquía 1 y por lo tanto presentan el mismo cómputo de valor jerárquico. No obstante, sobresale el valor jerárquico de los atractivos a escala local-comarcal, es decir de la jerarquía 2, al representar 180. En cuanto a las jerarquías con mayor valor jerárquico por recurso, en lo que respecta a la jerarquía 3 , ofrece un valor de 27 que se corresponde al Arco Romano y el yacimiento arqueológico del Tossal de Mortòrum, ambos en Cabanes, y el Castillo de Vilafamés. Por su parte, la jerarquía 4 se halla representada con un valor jerárquico de 16 en la Vía Augusta. Por último, es de destacar la jerarquía 5 ya que alcanza el valor jerárquico de 50 dada la potencialidad turística del Conjunto Histórico de Vilafamés y el Museo de Arte Contemporáneo Vicente Aguilera Cerni (MACVAC) del mismo municipio. 
En la categoría $C$, el valor de la jerarquía 1 es testimonial ya que solamente se halla representado por un recurso. De ahí que adquiere mayor protagonismo el valor jerárquico de la jerarquía 2 con una valoración de 20 pero, sobre todo, es la jerarquía 3 , con un valor de 63 quien define el potencial turístico de este categoría, expresado en los recursos vinculados por una parte a las bodegas de vino de Benlloch, Cabanes, Vilafamés, Vall d'Alba, y la Torre d'en Domènec, y por otra a la producción de turrones en Benlloch y Cabanes.

Por último, en la categoría $\mathrm{D}$, el valor jerárquico se halla repartido entre las jerarquías 1 con un valor de 21 , la jerarquía 2 que representa un valor de 56 y la jerarquía 3 que se concreta en acontecimientos programados de gran singularidad como son la Mostra de la vida tradicional: Vilafamés 1900, en Benlloch la festa de la Berema y el Feslloch, o los vinculados a la práctica deportiva entre los que sobresale la Marató de Borriol (MABO).

\section{Cuadro 1 \\ DISTRIBUCIÓN DE LOS RECURSOS TURÍSTICOS SEGÚN CATEGORÍAS Y VALOR JERÁRQUICO}

\begin{tabular}{|c|c|c|c|c|c|c|}
\cline { 2 - 7 } \multicolumn{1}{c|}{} & \multicolumn{5}{c|}{ Jerarquía (Valor jerárquico) } & TOTAL \\
\hline CATEGORÍAS & $\mathbf{1 ( 1 )}$ & $\mathbf{2 ( 4 )}$ & $\mathbf{3 ( 9 )}$ & $\mathbf{4 ( 1 6 )}$ & $\mathbf{5 ( 2 5 )}$ & \\
\hline A & $10(10)$ & $27(108)$ & $3(27)$ & $4(64)$ & $1(25)$ & $45(234)$ \\
\hline B & $30(30)$ & $45(180)$ & $3(27)$ & $1(16)$ & $2(50)$ & $81(303)$ \\
\hline C & $1(1)$ & $5(20)$ & $7(63)$ & 0 & 0 & $13(84)$ \\
\hline D & $21(21)$ & $14(56)$ & $4(36)$ & 0 & 0 & $39(113)$ \\
\hline TOTAL & $62(62)$ & $91(364)$ & $17(153)$ & $5(80)$ & $3(75)$ & $178(734)$ \\
\hline
\end{tabular}

\section{Categorías}

A. Atractivo o recurso natural-paisajístico

B. Recurso histórico-monumental, técnico, etnológico y artístico

C. Recurso artesanal y gastronómico

D. Folclore, fiestas y acontecimientos programados
Jerarquías

1. Complementa a otros recursos

2. Motiva corrientes turísticas locales

3. Motiva corrientes turísticas regionales

4. Atractivo a nivel nacional

5. Atractivo a nivel internacional

( ) Valor jerárquico, que se obtiene de multiplicar el número de recursos por el cuadrado de la jerarquía obtenida Fuente: López (2014) y Elaboración propia

Los resultados globales de la jerarquización de los recursos turísticos muestran que aquéllos que motivan corrientes turísticas locales constituyen el mayor número, seguidos por los que tienen un atractivo a nivel regional, aunque con un valor más bajo, precisamente en estos últimos son los recursos de categoría C. De entre los recursos con un grado de atracción turística a nivel nacional sobresalen los de la categoría A. En lo que respecta a aquéllos que ofrecen el mayor grado de atracción turística, representados en la jerarquía 5, corresponde a la categoría de recursos histórico-monumentales y a la categoría de recursos naturales-paisajísticos. 
Con la finalidad de corroborar estos resultados, en el cuestionario aplicado al panel de expertos, se ha contemplado una pregunta dirigida a valorar la potencialidad turística que presentan los recursos turísticos. Sobresale su valoración ya que obtiene el máximo acuerdo con las afirmaciones del cuestionario, la cual se traduce sobre todo en el potencial turístico de los recursos histórico- monumentales y etnológicos, y de los recursos paisajísticos y de observación, al ser los mejor valorados (4,6 respectivamente sobre un máxima de 5), seguido con un valor de 4,5 por el paisaje urbano tradicional de los núcleos de población, la gastronomía y el folclore, fiestas, acontecimientos programados e intangibles (Figura 2).

\section{Figura 2}

\section{VALORACIÓN DE LOS RECURSOS TURÍSTICOS}

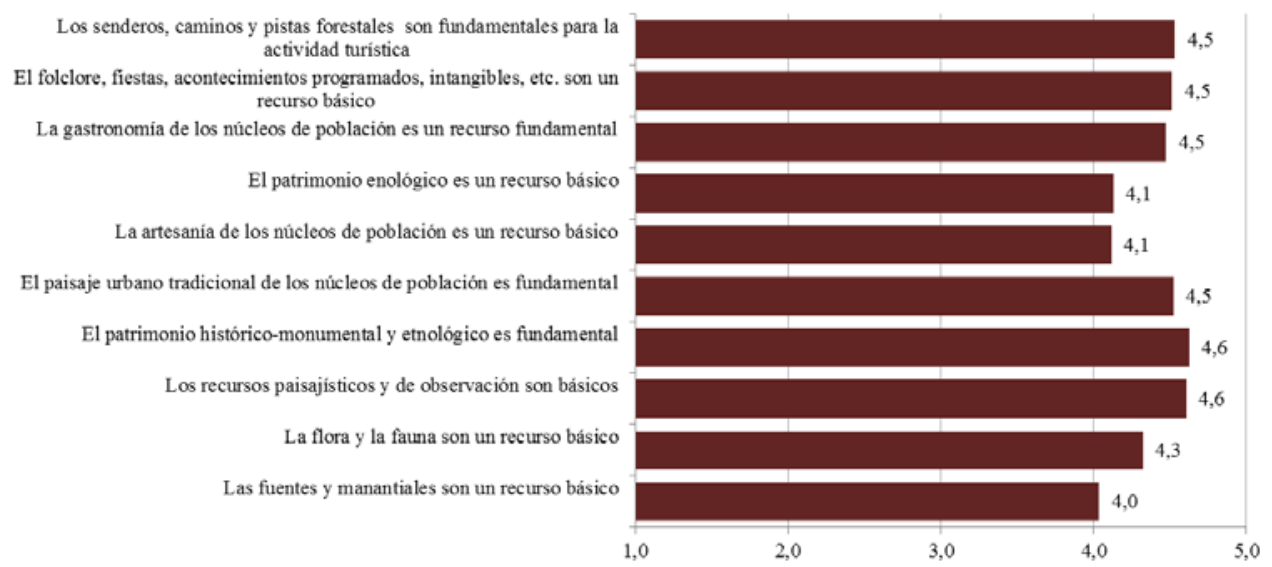

Fuente: Elaboración propia

\section{b) Las empresas}

Respecto a las empresas relacionadas con la actividad turística, el análisis se ha aplicado a la oferta de alojamiento comercial, tanto hotelero como extrahotelero, y al alojamiento residencial-turístico. A tenor de ello y tomando como referencia el periodo comprendido entre los años 2000 y 2015, se puede afirmar que el comportamiento de la oferta hotelera parte de una situación débil ya que en el total de los nueve municipios solamente existían 4 establecimientos que se correspondían a la categoría baja, de 1 y 2 estrellas, con capacidad de 91 plazas. Estas han experimentado una variación absoluta interanual expresada en un escaso incremento, ya que si bien la apertura de tres nuevos establecimientos supone duplicar la capacidad alojativa, éste solamente alcanza las 173 plazas, que siguen perteneciendo a las dos categorías inferiores, lo cual denota un acusado déficit.

En relación al alojamiento extrahotelero, las pensiones, tipología de alojamiento que se contempla en las estadísticas a partir de 2010, se reduce en ese año a un solo establecimiento con capacidad de 25 plazas, y en 2015 a 2 pensiones con un total de 31 plazas. 
En cuanto a los apartamentos turísticos, en el año 2000 esta tipología solamente se ofertaba en el municipio de Borriol, cuyos 20 apartamentos computaban 80 plazas. Su evolución ha sido positiva hasta alcanzar en el año 2015 un total de 48 apartamentos y 195 plazas, concentradas en los municipios de Borriol y Cabanes, ya que albergan el $72.9 \%$ de los apartamentos turísticos.

El alojamiento en campings es la tipología que representa el mayor número de plazas de la oferta alojativa del área de estudio, con 4 establecimientos ubicados todos ellos en la Ribera de Cabanes. Se mantiene el mismo número de establecimientos entre los dos años de referencia -2010 y 2015- pero la capacidad se ha incrementado en 520 plazas, pasando de 3.162 en el año 2000 a 3.682 en 2015. Cabanes se consolida en el cuarto destino de la provincia de Castellón en relación a esta tipología de establecimientos de alojamiento extrahotelero, siendo el único en disponer de un establecimiento de 5 estrellas.

Por último, las casas rurales y albergues representan la tipología con mayor dinamismo territorial en el área de estudio ya que mientras en el año 2000 solamente se ofertaba una casa rural en Vall d'Alba con 6 plazas, en el año 2015 se conforma en 20 casas rurales, con una capacidad total de 160 plazas. La oferta alojativa se completa con un albergue de 20 plazas.

El comportamiento del componente residencial queda expresado en la tasa de función turístico-residencial ya que determina la relación entre el número de viviendas secundarias y principales, y permite conocer el grado de especialización de un determinado espacio de destino turístico derivado del desarrollo residencial vinculado a la actividad turística. Tomando como valor de referencia 90, si el resultado del índice es superior a este valor indica una mayor especialización del destino en la componente residencial de la actividad turística, por el contrario, si no lo alcanza denota que el desarrollo residencial presenta un peso poco relevante en el sistema turístico del destino.

Así, considerando los datos del último Censo de Población y Viviendas (2011), una vez aplicada la tasa de función turístico-residencial a los municipios que integran el área de estudio, en general presenta un escaso potencial en oferta de alojamiento no comercial vinculada con las segundas residencias. Este hecho se explica al considerar que buena parte de los municipios no alcanzan o apenas logran sobrepasar la mitad del valor de referencia de 90, es el caso de Vall d'Alba (14), Sant Joan de Moró (25), Benlloch (26), la Pobla Tornesa y Vilafamés, ambos con un valor de 44, Cabanes (46) y Borriol (47). Más cercano al valor de referencia de 90 se encuentran Vilanova d'Alcolea (62) y solamente lo sobrepasa con un valor de 113 la Torre d'en Doménec.

Estos resultados han sido contrastados por medio del cuestionario aplicado al panel de expertos a través de una pregunta relacionada con la oferta turística (Figura 3). En ella se incluía, además de la valoración de la oferta de alojamiento, elementos de oferta complementaria y de gestión turística que repercuten sobre la rentabilidad y el dinamismo del alojamiento comercial y residencial-turístico. La valoración ha sido baja en la calidad del alojamiento, la débil estructuración de productos, así como la oferta comercial, excepto en el apoyo que se recibe desde el sector público. 


\section{Figura 3 \\ VALORACIÓN DE LA OFERTA TURÍSTICA}

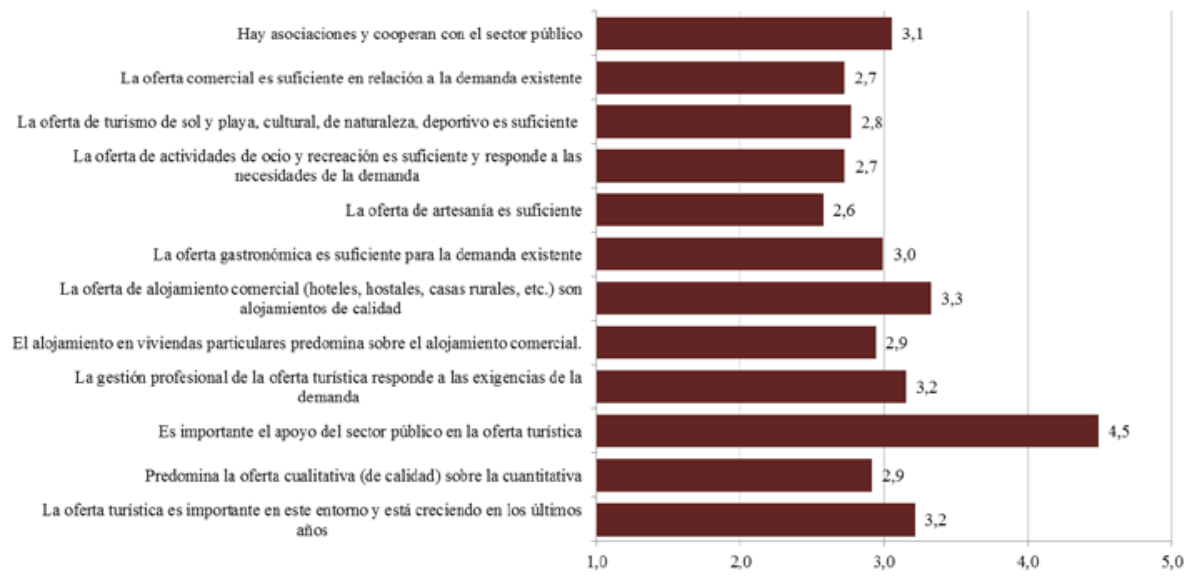

Fuente: Elaboración propia.

\section{c) Demanda}

El análisis de la demanda se ha efectuado a través de la explotación de los datos estadísticos elaborados por la oficina de turismo de Vilafamés correspondientes a los años 2015 y 2016. Así, comparando ambos años, presenta un balance positivo, ya que de los 4.595 visitantes de 2015 se ha pasado a 6.747 en 2016, lo que supone una variación interanual positiva del 46,8\%. En este comportamiento ha influido el hecho de que este destino se haya integrado, en la red de los Pueblos Más Bonitos de España. Paralelamente este reconocimiento también ha podido influir en reducir la estacionalidad, ya que en 2016 los meses de menor afluencia de visitantes han sido enero y febrero (548 y 495, respectivamente), y junio y julio con 504 y 498 visitantes.

Por otra parte, al objeto de concretar de manera específica la demanda internacional, se han considerado los resultados de un estudio sobre el perfil del turista internacional que accede vía aeropuerto de Castellón, de la Agència Valenciana del Turisme elaborado a finales de 2015 por la empresa Empymer (Agència Valenciana del Turisme, 2016). Los resultados manifiestan, atendiendo a las características socio-demográficas, se trata de un turista, de unos 50 año, está casado o que vive en pareja $(74,89 \%)$, tiene estudios superiores $(67,59 \%)$, está en activo $(59,40 \%)$ o jubilado $(32,52 \%)$ y pertenece a una clase social media $(40,82 \%)$ o media-alta $(40,15 \%)$. En cuanto a su comportamiento de compra y consumo cerca del $43 \%$ viaja en pareja mientras que algo más del $22 \%$ lo hace en familia, un $12,5 \%$ con amigos y el $21,5 \%$ lo hacen solos. Con respecto al motivo del viaje, en el $63 \%$ de los casos se realiza por motivos relacionados con el ocio y las vacaciones. Más concretamente, los motivos de la visita, además de disponer de una vivienda en propiedad o de amigos o familiares y venir a visitarlos, son: el clima; el precio; la comodidad del vuelo y porque buscan descansar o gozar de tranquilidad en espacios no masificados. 
Este último aspecto facilitará trabajar el posicionamiento del área de estudio, o lo que es lo mismo, su ventaja competitiva sostenible. Es decir, aquello que lo identifique como único y que, por tanto, lo haga diferente a los ojos de los demás, resulte difícil de imitar y perdure en el tiempo.

En lo que respecta a la estancia media, los turistas mayores de 65 años son los que realizan una estancia más larga, una media de 17 noches, mientras que el segmento de edad entre 45 y 64 años se caracteriza por una estancia media de 11 noches, y los menores de 45 años realizan una estancia más corta, de tan solo una semana de media.

En cuanto al lugar de pernoctación, solo uno de cada tres turistas lo hace en un alojamiento de pago, ya sea en hotel, aparta-hotel o apartamento turístico. La mayoría de los turistas se alojan en casa de amigos o familiares (36\%) o en su propia vivienda (30\%). Además, la estancia media en alojamiento de no pago (entre 13 y 14 noches) duplica a la estancia media en alojamiento de pago (entre 6 y 7 noches).

Finalmente, es importante señalar que las principales actividades que realizan son: ir de compras, visitar otros destinos, visitar amigos o familiares, disfrutar de la playa, ocio nocturno y hacer visitas culturales y actividades gastronómicas.

En cuanto a cómo conocieron el destino Comunitat Valenciana, cerca del $66 \%$ lo hicieron a través de recomendaciones de familiares y/ o amigos. Y uno de cada cinco (sobre todo en la franja de 25 a 44 años) lo conoció a través de internet y se apoyó en esta herramienta para organizar su viaje. En este sentido, cabe señalar que apenas un $1 \%$ contrató un paquete vacacional con un coste medio de 168,86 euros mientras que el 99\% programó el viaje por su cuenta y su gasto medio diario fue de 45 euros por persona.

\section{Figura 4}

\section{VALORACIÓN DE LA DEMANDA TURÍSTICA}

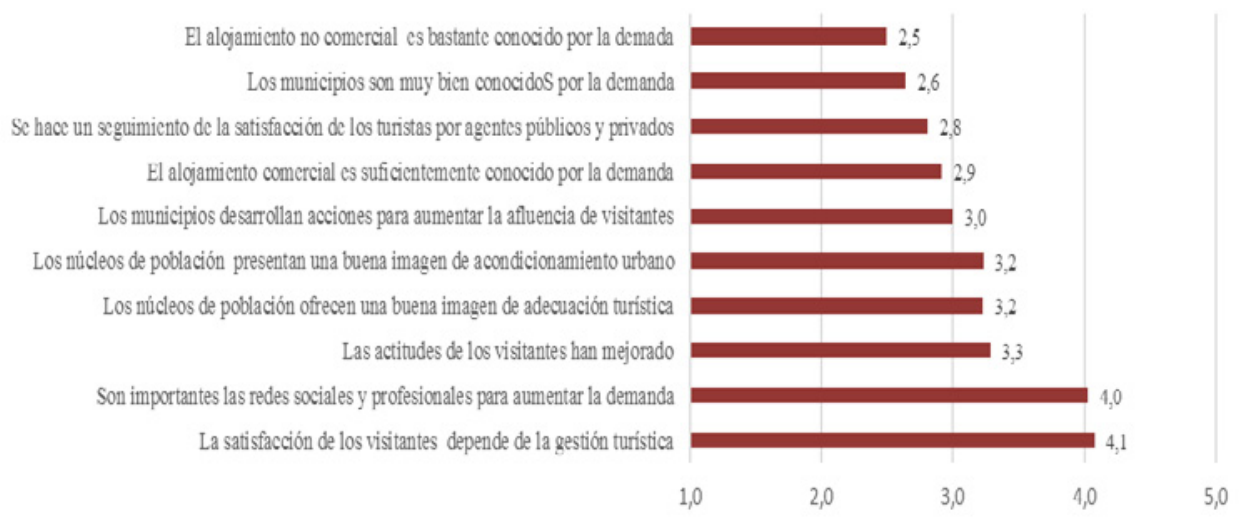

Fuente: Elaboración propia.

Así, al objeto de contrastar estos resultados con los del cuestionario, aplicado al panel de expertos, en los municipios del área de estudio, en aquél se ha contemplado una pregunta específica articulada en 10 ítems (Figura 4), con la finalidad de valorar el grado de 
adecuación de los municipios a las expectativas y exigencias de la demanda. Los resultados obtenidos en preguntas acerca del conocimiento de la demanda, de la identificación del producto turístico y del alojamiento comercial y no comercial, y el seguimiento de su comportamiento por parte de agentes turísticos públicos y privados, ofrecen una posición inferior a la media de valor 3 sobre un máximo de 5, dado que los municipios no son muy bien conocidos por la demanda; tampoco ofertan productos turísticos bien identificados; el alojamiento comercial (hoteles, hostales, casas rurales, etc.) no es suficientemente conocido, al igual que el alojamiento no comercial; y la satisfacción de los turistas se viene siguiendo poco por parte de los agentes turísticos privados y públicos.

Tan sólo en las preguntas acerca de la importancia de las redes sociales y ferias para favorecer el conocimiento de la demanda, así como de la relación entre la satisfacción de los visitantes y la gestión turística, y acerca de la afirmación de que la actitud de los visitantes ha mejorado, no registran valores por debajo de la media, habiendo así consenso al haber valorado positivamente las afirmaciones acerca de la importancia del apoyo del sector público en la generación de oferta; la importancia de las redes sociales, profesionales y las campañas en ferias para la captación de visitantes; o en la percepción que las actitudes de los visitantes han mejorado.

\section{d) Infraestructuras y servicios}

El análisis de las infraestructuras y servicios se ha realizado a partir de la valoración efectuada por el panel de expertos en los cuestionarios, en lo que se refiere al análisis de la situación del sistema turístico y al estudio prospectivo.

En primer lugar sobresalen con una valoración positiva, superior a la media de 3, además de la propia infraestructura aeroportuaria, el sistema de recogida de residuos sólidos; la red de abastecimiento de agua potable; la accesibilidad en vehículo propio a los municipios; el tratamiento de las aguas residuales; la calidad del agua; y la red de suministro eléctrico. Por su parte, se sitúan en el valor medio de 3 , las preguntas referidas a la conectividad externa; la cobertura telefónica móvil; acceso a redes telemáticas de alta capacidad; el estado de conservación de vías pecuarias, caminos y senderos. Por el contrario la oferta formativa dirigida a los profesionales de servicios turísticos, es la que presenta un valor inferior a la media (Figura 5).

Los destinos que registran mayor proporción de valores máximos, es decir 5, son Vilafamés y Cabanes, seguido en menor proporción de la Pobla Tornesa y Sant Joan de Moró, éste último presenta la mayor distribución de valor 4. Cabanes, además de registrar la segunda mayor proporción de valores 5, es el municipio que también concentra el mayor número de valoraciones más bajas, a los que le siguen a mayor distancia Vilafamés, Vilanova d'Alcolea y Vall d'Alba.

Este apartado se completa con la pregunta acerca de "Las nuevas tecnologías y la gestión de la información“. Ésta presenta valores medios muy por debajo de la media en las subpreguntas acerca de si han contado con gran ayuda en la utilización del sistema, de la afirmación de que cuentan con los recursos necesarios para utilizar el sistema informático para la gestión de la información y que cuentan con ayuda para prestar asistencia con las dificultades del sistema. Es de remarcar que en el ámbito empresarial como a nivel de 
destino, las nuevas tecnologías y la gestión de la información (Figura 6) desempeñan un papel importante en la actividad turística, coincidiéndose en que el sistema informático es útil para el trabajo. Por el contrario, se obtienen resultados menos satisfactorios al considerar que no se dispone de persona o grupo específico para prestar asistencia con las dificultades del sistema y que no se utilizan suficientemente las nuevas tecnologías como herramientas de comunicación. Se puede afirmar que los responsables de los destinos turísticos son conscientes de la importancia que tienen actualmente las tecnologías de la información y que deberían destinar mayores recursos para una utilización eficaz con el fin de explotar el potencial que actualmente tienen las TIC para la mejora de la competitividad de los destinos turísticos.

\section{Figura 5 \\ VALORACIÓN DE LAS INFRAESTRUCTURAS Y SERVICIOS}

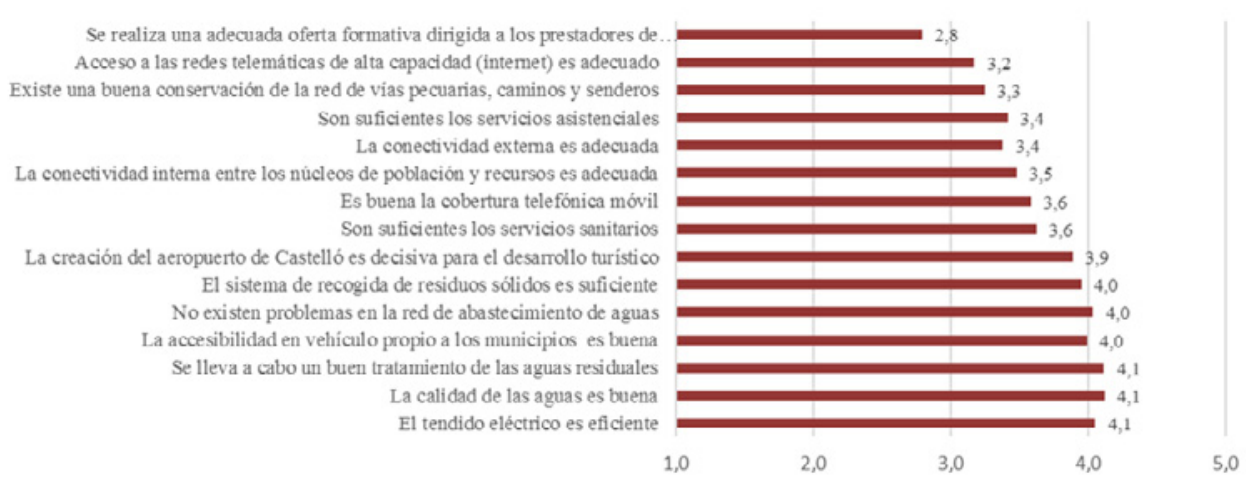

Fuente: Elaboración propia.

Figura 6

LAS NUEVAS TECNOLOGÍAS Y LA GESTIÓN DE LA INFORMACIÓN

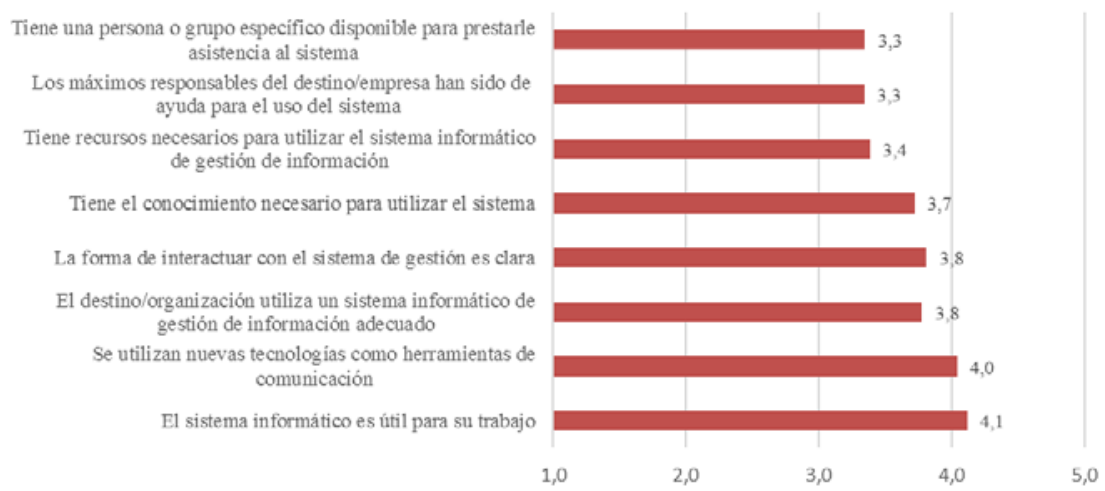

Fuente: Elaboración propia. 


\section{EXPLOTACIÓN GLOBAL DE RESULTADOS DEL CUESTIONARIO TIPO DELPHI APLICADO A UN PANEL DE EXPERTOS}

En la aplicación de la escala Likert 5 los valores globales medios obtenidos en el cuestionario se aproximan a 4. Por municipios, la media más alta la registra en Sant Joan de Moró (4.4) y Vilafamés (4.1), seguido de valores muy próximos en los municipios de Vall d'Alba, Vilanova d'Alcolea y la Torre d'en Doménec (3.6 en los tres casos).

Estableciendo como umbral el valor inferior a 3 y superior a 4 se pueden identificar dos grupos de ítems o afirmaciones a nivel supramunicipal, en los que hay consenso en todos los municipios. El primero expresa los valores superiores a la media, es decir, los que superan el valor 4 (Cuadro 2) y el segundo los valores inferiores a la media, es decir, menores al valor 3 (Cuadro 3 ).

Los valores medios, a nivel supramunicipal, revelan estar de acuerdo con las afirmaciones de que los recursos paisajísticos, el patrimonio, y el paisaje tradicional urbano son necesarios para el desarrollo turístico. Los valores máximos se registran en la pregunta de los "Recursos Turísticos" y la "Demanda Turística" (Cuadro 2). En general, el panel de expertos manifiesta su máximo acuerdo con los recursos turísticos, que son fundamentales para el desarrollo turístico, la buena cobertura y calidad de las infraestructuras y servicios, la accesibilidad y el conocimiento de las nuevas tecnologías para la gestión turística, y en relación con el desarrollo turístico sostenible.

\section{Cuadro 2}

PREGUNTAS CON VALORES SUPERIORES A LA MEDIA (>4)

\begin{tabular}{|c|c|}
\hline Recursos Turísticos & Demanda Turística \\
\hline $\begin{array}{l}\text { - La importancia de los recursos turísticos } \\
\text { para el desarrollo turístico, considerando en } \\
\text { menor medida la importancia de las fuentes } \\
\text { y manantiales como un recurso básico para } \\
\text { el desarrollo turístico (4.0). } \\
\text { - Los recursos paisajísticos y de observación } \\
\text { son básicos para el desarrollo turístico de los } \\
\text { municipios (4.6) } \\
\text { - El patrimonio histórico-monumental y et- } \\
\text { nológico es fundamental para el desarrollo } \\
\text { turístico (4.6) } \\
\text { - El paisaje urbano tradicional de los núcleos } \\
\text { de población es fundamental para el desarro- } \\
\text { llo turístico (4.5) }\end{array}$ & 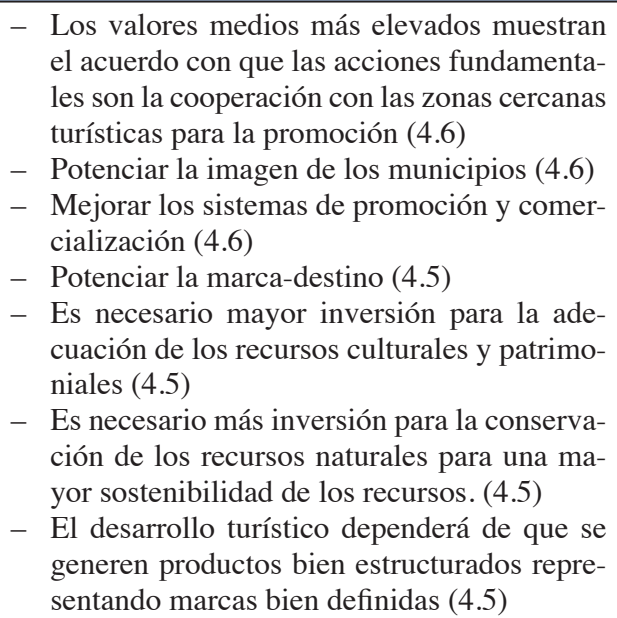 \\
\hline
\end{tabular}

Fuente: Elaboración propia.

Por el contrario, casi todos ítems de las preguntas "Oferta Turística" y "Demanda Turística" se registran valores inferiores a la media (Cuadro 3). Tan solo en las subpregun- 
tas acerca del apoyo del sector público en la oferta turística, la importancia de las redes sociales y profesionales y campañas en ferias para la captación de la demanda, la importancia de la gestión turística para la satisfacción de la demanda y la afirmación acerca de que las actitudes de los visitantes han mejorado, no se registra algún valor por debajo de la media. Por lo que se refiere a la pregunta sobre la "Promoción, comercialización, financiación y gestión pública", esta registra un elevado número de valores inferiores a la media a nivel municipal. La media más baja de esta pregunta se obtiene en la subpregunta acerca de la existencia de una política de promoción eficiente. Esta valoración se registra en Cabanes, uno de los municipios más críticos en materia de promoción, comercialización, financiación y gestión pública, seguido de Vilanova d'Alcolea, la Torre d'en Doménec, Vall d'Alba, Benlloch y la Pobla Tornesa.

\section{Cuadro 3}

\section{PREGUNTAS CON VALORES INFERIORES A LA MEDIA $(<3)$}

\section{Oferta Turística}

- Predomina la oferta cualitativa (de calidad) sobre la cuantitativa (2.9)

- El alojamiento en viviendas particulares predomina sobre el alojamiento comercial (2.9)

- La oferta de artesanía es suficiente (2.6)

- La oferta de actividades de ocio y recreación es suficiente y responde a las necesidades y preferencias de la demanda (2.7)

- La oferta de turismo cultural, enológico, de naturaleza, deportivo es suficiente en los municipios. (2.8)

- La oferta comercial es suficiente en relación a la demanda existente (2.7)

- Existen suficientes puntos de información (2.0)

- Existe una ruta estructurada y conocida por los visitantes (2.0)

- La señalización de los recursos turísticos es adecuada (2.2).

Promoción, comercialización, financiación y gestión pública

- Los productos turísticos se comercializan bien (2.9)

- La administración central y sus programas de política turística han facilitado el desarrollo de la oferta y de la actividad turística (2.9)

- Los análisis y estudios que se realizan en torno a los municipios son suficientes y prácti$\cos (2.5)$

\section{Demanda Turística}

- Los municipios son muy bien conocidos por la demanda (2.6)

- Los municipios tienen productos turísticos bien identificados (2.6)

- El alojamiento comercial (hoteles, hostales, casas rurales, etc.) es suficientemente conocido por la demanda (2.9)

- El alojamiento no comercial se considera una modalidad bastante conocida por la demanda (2.5)

- La satisfacción de los turistas se viene siguiendo por parte de los agentes turísticos privados y públicos (2.8)

Fuente: Elaboración propia. 
Por último, en relación a las preguntas abiertas el análisis permite identificar:

1. Falta de una estrategia turística global para el conjunto de municipios.

2. No existe un producto turístico integrado. Los diferentes encuestados proponen el diseño de rutas a pie y en bici, y la puesta en valor de recursos.

3. Unido a lo anterior, inexistencia de una imagen de marca.

4. Falta de iniciativas privadas y falta de consenso de éstas con la administración pública local.

5. Necesidad de un órgano de gestión turística comarcal.

6. La necesidad de mejorar la infraestructura y los servicios turísticos, entre otros, mejorar la señalización. En este aspecto reclaman más intervención por parte de las instituciones públicas.

7. Es destacable la falta de credibilidad e implicación de la población residente en relación al desarrollo de la actividad turística, a pesar de que los agentes sociales encuestados, en la pregunta 15 , acerca del nivel de desarrollo frente a sus competidores, afirman ser conscientes del potencial turístico que tienen y de las oportunidades económicas que puede traer el turismo a la población. En este sentido, hay que señalar que en la pregunta 11, acerca de las tendencias y cambios que se pueden producir en el entorno del aeropuerto, se detecta una visión economicista del turismo, en la que abogan por el incremento de rentas, la creación de empleo y el estímulo que suponen estos beneficios para el buen "clima" turístico por parte de la población.

\section{DIAGNÓSTICO Y PROPUESTA DE DESARROLLO TURÍSTICO INTEGRADO}

Tras el análisis de los elementos del sistema turístico, los resultados de los cuestionarios aplicados al panel de expertos, y los resultados en la I y II Jornada de Sensibilización, con la presencia de los representantes políticos municipales de los municipios y representantes de la Agència Valenciana del Turisme, se consensuaron los principales resultados del diagnóstico (Cuadro 4).

\section{Cuadro 4 \\ MATRIZ DAFO}

\section{Fortalezas}

- Los recursos paisajísticos y de observación

- Espacios naturales protegidos (Paraje Natural del Desert de les Palmes, Parque Natural del Prat de Cabanes-Torreblanca, Parajes naturales municipales El Mollet y San Miguel)

- La playa de Torre la Sal de la Ribera de Cabanes (granulometría baja)

- El patrimonio histórico-monumental y etnológico. Vía Augusta y Arc de Cabanes

- El paisaje urbano tradicional. BIC Vilafamés

- El folclore, las fiestas y los acontecimientos programados

- La gastronomía

- Los productos agroalimentarios (Almendras, vino, aceite)

- El Museo de Arte Contemporáneo de Vilafamés

- Buena conexión con la CV-10. Buena accesibilidad excepto algunos puntos desde la costa 
- El aeropuerto de Castellón

- Buenas condiciones climáticas (favorecen el ocio, la recreación y el turismo)

- La homogeneidad de este espacio entorno al Pla de l'Arc

- Proximidad al área turística del litoral de Castellón

\section{Debilidades}

- Faltan criterios en el uso del suelo que pueden producir disfunciones territoriales y turísticas

- Municipios con población envejecida

- Falta de productos bien estructurados. Deficiencias en la puesta en valor de los recursos

- Predomina la oferta cuantitativa sobre la cualitativa

- Débil oferta artesanal

- Insuficiente oferta de ocio y recreación

- Insuficiente oferta de alojamiento comercial (hoteles, hostales, etc.)

- Falta de asociacionismo empresarial

- Los municipios no son muy conocidos por la demanda

- El alojamiento comercial no es muy conocido

- Ausencia de alojamientos hoteleros de calidad media (3 estrellas) y de lujo (hoteles de 4 y 5 estrellas)

- Déficit en la señalización de los recursos

- Débil gestión y promoción turística

- Deficiente conexión ferroviaria

- Los transportes públicos y sus frecuencias

- Déficit en la utilización de las TIC

- Falta de relaciones supramunicipales

\section{Oportunidades}

- Aprovechar el potencial de los recursos turísticos y su transformación en productos

- El aeropuerto de Castellón

- La mejora de la accesibilidad a través de la CV-10

- Mejor aprovechamiento de los productos agroalimentarios

- Potenciar la gastronomía

- Incorporación de nuevos productos turísticos en base a las tendencias de la demanda, turismo ornitológico, senderismo, deporte, etc.

- Potenciar las conexiones costa-interior

- El incremento de la preocupación de las administraciones por la puesta en valor de los recursos y la comercialización de los productos

- Mejorar el desarrollo turístico en base a la nueva gobernanza público-privada, sobre todo a nivel supramunicipal y hacer identificable este espacio para la demanda

- Importante número de turistas en áreas limítrofes

- Posibilidad de explotar el concepto "exclusividad" (ausencia de oferta hotelera de 3, 4 y 5 estrellas)

\section{Amenazas}

- Alterar el paisaje ambiental y cultural

- El desarrollo adecuado de las industrias y su contaminación visual

- La contaminación acústica de ciertas poblaciones al paso de la CV-10

- El no poner en valor los recursos está perjudicando los valores intrínsecos de los recursos, sobre todo los patrimoniales y etnológicos

- Escasa tradición laboral en el sector turístico

- La competencia de otros espacios se convierte en una amenaza

Fuente: Elaboración propia. 


\section{DISEÑO DE LAS ESTRATEGIAS}

El diseño de las estrategias parte de la formulación de unos objetivos específicos, fruto de los resultados obtenidos en el diagnóstico expresado a través de la matriz DAFO. Dentro de estos se contemplan: los aspectos demográficos y socioeconómicos, medioambientales, recursos turísticos, alojamiento, oferta complementaria y de ocio, infraestructura y servicios, y promoción y comercialización.

Estos objetivos dirigen la creación de la propuesta de estrategias (Cuadro 5), entre las que es clave la estrategia dirigida a la generación de productos turísticos bajo enfoque supramunicipal y cuya base se encuentra en los propios recursos turísticos. Así, el área de estudio tiene potencialidad para desarrollar los siguientes productos: turismo de naturaleza en los espacios naturales protegidos, turismo cultural, el turismo activo, productos etnológicos, productos enológicos, turismo deportivo, productos de acontecimientos programados, turismo de golf y excursionismo.

Asimismo, se considera fundamental adecuar los productos turísticos a las motivaciones y exigencias reales de la demanda turística, por lo que se definieron dos perfiles de turistas atendiendo a aspectos sociodemográficos.

\section{Cuadro 5 \\ DISEÑO DE ESTRATEGIAS DE ÁMBITO SUPRAMUNICIPAL EN EL ENTORNO DEL AEROPUERTO}

\begin{tabular}{|c|c|c|c|}
\hline $\begin{array}{l}\text { Estrategia de calidad del } \\
\text { medio } \\
\text { Programas: } \\
\text { - Adecuación de las } \\
\quad \text { infraestructuras } \\
\text { - Medio ambiente } \\
\text { - Ordenación de las UAT }\end{array}$ & $\begin{array}{l}\text { Estrategia de productos } \\
\text { turísticos } \\
\text { Programas: } \\
\text { - Turismo verde y de } \\
\text { naturaleza. } \\
\text { - Turismo cultural y } \\
\text { activo. } \\
\text { - Producto etnológico. } \\
\text { - Producto enológico. } \\
\text { - Turismo deportivo. } \\
\text { - Acontecimientos } \\
\text { programados. } \\
\text { - Sol y playa individual. } \\
\text { - Turismo de golf. } \\
\text { - Excursionismo. }\end{array}$ & $\begin{array}{l}\text { Estrategia de } \\
\text { alojamiento, } \\
\text { restauración y } \\
\text { comercio. } \\
\text { - Programa de } \\
\text { creación de } \\
\text { alojamiento } \\
\text { hotelero. } \\
\text { - Programa de } \\
\text { alojamiento rural. } \\
\text { - Programa de } \\
\text { restauración. } \\
\text { - Programa sobre } \\
\text { comercios. } \\
\text { - Programa de nuevas } \\
\text { tecnologías TIC. }\end{array}$ & $\begin{array}{l}\text { Estrategia de } \\
\text { comercialización } \\
\text { - Programa de } \\
\text { promoción y } \\
\text { comercialización de } \\
\text { productos turísticos. } \\
\text { - Programa de } \\
\text { marketing } \\
\text { relacional. } \\
\text { - Programa de imagen } \\
\text { turística. }\end{array}$ \\
\hline $\begin{array}{l}\text { Estrategia de } \\
\text { dinamización turística } \\
\text { - Programa de servicios } \\
\text { públicos. } \\
\text { - Programa de agentes } \\
\quad \text { privados. } \\
\text { - Programa de formación. } \\
\text { - Programa de cooperación } \\
\text { y organización. }\end{array}$ & & & \\
\hline
\end{tabular}

Fuente: Elaboración propia. 
Por una parte, el perfil 1, conformado por un turista de mediana edad, de clase media-alta, que trabaja, vive en ciudad y en pareja y que no tiene hijos. En cuanto a sus hábitos como visitante, lo hace varias veces al año pero suelen ser estancias cortas (lo que se conoce como escapadas) y siempre lo hace en pareja. Además, gestiona el viaje por su cuenta, casi siempre online y no busca precio. Al contrario, es un turista preocupado por la calidad que desea analizar los productos turísticos con tiempo y que da mucha importancia a poder realizar itinerarios flexibles, es decir, viajes con una alta dosis de espontaneidad. Finalmente, en relación a su motivación para viajar, lo hace por el interés de conocer nuevos lugares, de poder aprender y de poder encontrar experiencias auténticas y sobre todo, significativas para él.

En cuanto al perfil 2 está formado por turistas de edad más avanzada y que pese a que en términos de ingresos, dado que ya no trabaja, formaría parte de una clase media, su estilo de vida hace que su nivel de gasto en actividades de ocio y recreación sea alto. En este sentido, también viaja varias veces al año y las estancias suelen ser más largas. Se decantan mayoritariamente por viajes en los que disfrutar de la gastronomía, de los paisajes, de la cultura, entre otros, ya que su edad ilustra a un viajero con ganas de disfrutar y por tanto, va a querer experimentar e interactuar con el destino. Además, en relación a la planificación del viaje, huye de todo lo que suena a paquete turístico y por tanto, suele contratar directamente en destino, sin la intermediación de las agencias de viaje.

\section{CONCLUSIONES}

Se puede afirmar que el objetivo general de la investigación se ha alcanzado tanto en su análisis interno como externo, conociendo el posicionamiento de los municipios del entorno del aeropuerto de Castellón en los aspectos socio-económicos, a la vez que su predisposición para un mayor desarrollo de la actividad turística, actividad que tiene mucha posibilidad de desarrollarse de manera estructurada, como se ha podido deducir de la potencialidad que alberga en base a los recursos turísticos. Además y desde una perspectiva territorial, el enfoque supramunicipal ha tenido muy buena acogida entre los nueve municipios que conforman el área de estudio, tanto entre el sector público como en el privado, lo cual es importante remarcar para la sostenibilidad del desarrollo turístico.

A partir de los resultados del análisis aplicado a los elementos del sistema turístico integrado, las entrevistas al panel de expertos y el diagnóstico, hemos podido reflejar una serie de potencialidades, que se han podido expresar en la propuesta de estrategias, traducidas básicamente en los recursos turísticos que atesora el área de estudio. Pero a su vez han dejado claras unas debilidades importantes como es la falta de estructuración de productos turísticos. Precisamente las debilidades, con la excepción de los recursos, han quedado patentes en la oferta de alojamiento reglado, los medios de transporte públicos, servicios o falta de inversiones, entre otros aspectos que se han identificado en el diagnóstico. Sin embargo, la puesta en funcionamiento del aeropuerto y considerando las actuales tendencias de la nueva demanda, ambos se presentan como oportunidades que pueden y deben ser aprovechadas por los municipios del entorno de esta infraestructura. 
Por ello, los objetivos deben en primer lugar incidir en fortalecer los aspectos básicos demográficos y socio-económicos, sobre todo en una mayor coordinación y asociacionismo empresarial, y en una mayor sensibilización de la sociedad civil; así como en la calidad del medio, lo cual conlleva un componente mucho más transversal al estar orientados hacia la sostenibilidad del territorio, traducida en la línea de actuar sobre el tratamiento de residuos derivados de actividades ganaderas, en los impactos visuales en el paisaje y en la capacidad de carga.

Ya dentro del sistema turístico, en el caso específico de los recursos turísticos, los objetivos deben incidir en la puesta en valor del potencial turístico del patrimonio natural, paisajístico y cultural, la gastronomía, y los acontecimientos programados, para la creación de productos competitivos. También es clave el objetivo de fortalecer la oferta de alojamiento reglado, ya que este presenta una gran debilidad tanto en número de plazas como desde un criterio cualitativo en la diversificación de categorías, puesto que solamente destacan las plazas de camping ubicadas en la franja litoral de Cabanes. Por lo tanto, para generar dinámica de producto que mejore el posicionamiento del área de estudio en el mercado turístico, es necesario incrementar el número de establecimientos hoteleros y además potenciar su calidad. En este mismo sentido, la aplicación de nuevas tecnologías se presenta como un objetivo primordial, dada la debilidad que presenta el área de estudio sobre todo a nivel empresarial en la comercialización de la oferta de alojamiento reglado, restauración y comercio. En relación a la demanda, los objetivos se encaminan a mejorar el posicionamiento del área de estudio en el mercado, ya que no se ofertan productos turísticos bien identificados; el alojamiento comercial (hoteles, hostales, casas rurales, etc.) no es suficientemente conocido, al igual que el alojamiento no comercial; y la satisfacción de los turistas se viene siguiendo poco por parte de los agentes turísticos privados y públicos. Igualmente como objetivo se plantea incrementar la oferta complementaria y de ocio, caso de red de senderos. En materia de infraestructuras, considerando el posicionamiento territorial del aeropuerto de Castellón en el área de estudio, se fija como objetivo mejorar la conexión viaria de este espacio de interior con la costa, al igual que mejorar el transporte público e incrementar los servicios de información. También potenciar e impulsar la promoción turística en base a la potencialidad de productos que el área de estudio puede generar. Como se ha contemplado, éstos deben diseñarse a través de una estrategia específica de productos turísticos que desarrollen: el turismo de naturaleza, el turismo cultural, el turismo activo, el etnológico, el turismo enológico, el turismo deportivo, el turismo de golf y el excursionismo, complementada con una estrategia de comercialización.

Por último, y enfocado hacia la gobernanza y la participación en la gestión turística sostenible, se ha propuesto una estrategia de dinamización articulada en programas dirigidos en primer lugar a fortalecer los servicios públicos; a sensibilizar a los agentes privados implicados en el sistema turístico del área de estudio; un tercer programa dirigido a la formación sobre todo de los agentes sociales que participan en la actividad turística; y un programa de cooperación y organización en el cual se integra a la sociedad civil de los municipios del entorno del aeropuerto de Castellón. 


\section{BIBLIOGRAFÍA}

AGÈNCIA VALENCIANA DEL TURISME (2016): Perfil del turista internacional que visita la Comunitat Valenciana vía aeropuerto de Castellón. Generalitat Valenciana. Valencia.

BESANCENOT, J-P. (1991): Clima y Turismo. Barcelona. Masson.

GARROD, B., y FYALL L, A. (2005): "Revisiting Delphi: The Delphi Technique in tourism research", en Tourism Research Methods (Ritche, B., Burns,P. y Palmer, C. (Eds.), CAB International.

LINSTONE, H.A y TUROFF, M. (Eds.) (2002): The Delphi Method: Techniques and applications. Addison-Wesley Publising.

LÓPEZ, D. (1999): "Las tendencias de la demanda y las políticas turísticas integradas, como instrumentos de revitalización de los espacios rurales españoles de interior". Boletín de la Asociación de Geógrafos Españoles, $\mathrm{n}^{\circ}$ 28, pp. 61-83

LÓPEZ, D. (2003): "El desarrollo turístico en los espacios Rurales de interior: su aplicación al producto de salud". Cuadernos de Turismo, n 32, pp. 107-126.

LÓPEZ, D. (2014): Los recursos turísticos. Evaluación, ordenación y planificación turística. Estudio de casos. Valencia. Tirant lo Blanch.

MANERO, F. (1997): "Experiencias de turismo interior: logros y progresos desde la perspectiva del desarrollo local", en Los turismos de interior. Universidad Autónoma de Madrid. pp 207-329.

MATEU, J.F. (1982): El norte del País Valenciano. Geomorfología litoral y prelitoral. Valencia. Universidad de Valencia. Sección de Geografía.

MELLA, J.M. (1998): "Evolución doctrinal de la ciencia regional: una síntesis", en Economía política regional en España ante la Europa del siglo XX. Akal. Madrid.

MOUTINHO, L. y WITT, S.F. (1995): "Forecasting the tourism environment using a consensus approach". Journal of Travel Research $\mathrm{n}^{\circ} 33$ (4), pp 46-50.

NORTHCOTE J., LEE, D., CHOK, S. y WEGNER, A. (2008): "An Email-Based Delphi. Approach to tourism program evaluation: Current stakeholders in research design". Current Issues in Tourism $\mathrm{n}^{\circ} 11$ (3), pp. 296-297.

PULIDO, M. y PULIDO, J.I. (2016): “¿Existe un buen modelo para implementar la gobernanza en destinos turísticos? La opinión de los expertos en sostenibilidad en los modelos actuales de gestión turística" en Sostenibilidad en los modelos actuales de gestión turística (López, D., Edit.). Valencia. Tirant lo Blanch, pp. 57-85.

SEPE (2016): Informe del tejido empresarial de la provincial de Castellón. Información municipal 2015. Observatorio de las Ocupaciones del Servicio Público de Empleo Estatal. Dirección Provincial de Castellón.

SOLSONA, J. y LÓPEZ, D. (2012): "Factores de localización y desarrollo turístico en el espacio rural de la Comunitat Valenciana". Boletín de la Asociación de Geógrafos Españoles, $\mathrm{n}^{\circ}$ 59, pp. 221-243. 
